\title{
Variation of Conductivity with Temperature for the Normal State of Oxide Superconductors
}

\author{
R. J. Thorn \\ Materials Science/Chemistry Divisions \\ Argonne National Laboratory \\ Argonne, $\Pi 60439$
}

\section{DISCLAIMER}

This report was prepared as an account of work sponsored by an agency of the United States Government. Neither the United States Government nor any agency thereof, nor any of their employees, makes any warranty, express or implied, or assumes any legal liability or responsibility for the accuracy, completeness, or usefulness of any information, apparatus, product, or process disclosed, or represents that its use would not infringe privately owned rights. Reference herein to any specific commercial product, process, or service by trade name, trademark, manufacturer, or otherwise does not necessarily constitute or imply its endorsement, recommendation, or favoring by the United States Government or any agency thereof. The views and opinions of authors expressed herein do not necessarily state or reflect those of the United States Government or any agency thereof.

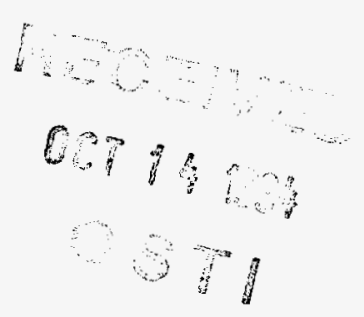

The submitted manuscript has been authored by a contractor of the U.S. Government under contract No. W-31-109-ENG-38. Accordingly, the U. S. Covemment retains a nonexclusive, royalty-free ficence to publish or reproduce the published form of this contribution, or allow others to do so, for U.S. Government purposes. 


\section{DISCLAIMER}

Portions of this document may be illegible in electronic image products. Images are produced from the best available original document. 


\begin{abstract}
Several equations have been proposed in the literature to describe the variation of resistivity with temperature, $\rho(T)$, for the superconducting oxides. Among these, a linear variation of $\rho(T)$ has been accepted as a characteristic feature for these materials. These equations have been compared through least squares' application to the experimental values of $\rho(T)$ for one case of $\mathrm{YBa}_{2} \mathrm{Cu}_{3} \mathrm{O}_{7-\delta}$ with $\delta$ near 0.1 . It has been found that two equations, (a) one based on an averaged density of state and small Fermi and thermal gap energies and (b) another based on Holstein's small polaron, yield the lowest percent deviations from the experimental values and the smallest chi squared/degree of freedom. They are also the only equations that describe quantitatively the variations with both temperature and composition.
\end{abstract}




\section{Introduction}

Recently several studies have recognized that the resistivity (conductivity) of the normal state of oxide superconductors is an interesting phenomenon and one that should be investigated as a basis for understanding the onset of the superconducting state. Among these studies, at least seven equations based on somewhat different theories have been used to describe $\rho(T)$, at least that of the optimum situation. During the same time, and in fact, as part of these studies the belief has evolved that $\rho(T)$ for the optimum case is linear with a high degree of precision (1-21). Unless all of these theories are essentially the same and the equations are various forms of one equation, it seems doubtful that they can all yield meaningful insights into the evolution of the superconducting state. Hence to ascertain their common feature, if any, their differences, and their validities, I have conducted a comparative study of them. The content of the study is not to dwell on the large variety of physical phenomena usually associated with each and used to establish its validity, but rather to examine crucial, phenomenological aspects and to attempt to identify any common conceptual features among them.

\section{Brief Descriptions}

The variously proposed equations are listed in Table I together with, where possible, the identification of the basic theories involved and references to the recent publications. In addition, brief descriptions are given of the conceptual bases, where it is possible to identify such. To accomplish this study, it is not necessary to delve into the details of each theory. Indeed, such is not possible in every case, because the theories are not extensively developed in some of the cases or at least not developed to the same extents. However, it is possible to identify in most cases the particularly characteristic features. 
Table I. Equations for Variation of $\rho(T)$ or $\sigma(T)$ for Normal State of Oxide Superconductors

\section{Equation}

1. $\rho=A T^{-1}+B T+C$

2. $\rho=\alpha \ln T+\beta T+\gamma$

3. $\rho=\rho o+K\left(\frac{T}{T_{f}}\right)^{2} \ln \left(\frac{T_{f}}{T}\right)$

4. $\sigma=\mathrm{CT}^{-1} \operatorname{sech}^{2}(\beta \mathrm{E})$

5. $\sigma=\sigma_{0}\left\{\left(e^{-\beta E_{f}}=1\right)-1-\left(e^{\beta\left(E_{z}-E_{\vartheta}\right)}+1\right)-1\right\}$

6. $p=C\left(e^{\beta E}+1\right)^{-1}$

7. $\sigma=C T^{1-n} \ln \left\{\frac{\left(e^{\beta E_{f}}+1\right)\left(e^{-\beta\left(E_{g}-E_{f}\right)}+1\right)}{2}\right\}$
Name

RVB

Kondo

Fermi Liquid

25,26

Phonon-coupled

Electron Exchange

$g(\varepsilon)=$ const for $0<\varepsilon<w$, otherwise zero

28

anharmonic/vibronic coupling

29

$$
\mathrm{g}(\varepsilon)=0 \text { for } \mathrm{E}_{\mathrm{f}}<\varepsilon<\mathrm{E}_{\mathrm{g}}
$$

and constant otherwise

30,31

8. $\sigma=\sigma_{\mathrm{h}}+\sigma_{\mathrm{t}}$

$$
\sigma=\mathrm{C}_{\mathrm{h}} \mathrm{T}^{-3 / 2} \mathrm{E}^{-1 / 2} \mathrm{e}^{-\beta E}+\mathrm{C}_{\mathrm{t}} \mathrm{T}^{-1}[\operatorname{csch} \beta \omega]^{1 / 2} \mathrm{e}^{-\mathrm{g} \operatorname{csch}(\beta \omega)} \quad \begin{aligned}
& \text { Small Polaron } \\
& \text { (Holstein) }
\end{aligned}
$$

$$
\beta=(k T)^{-1}
$$




\section{Resonance Valence Bond}

The RVB model has its origin in Pauling's description of a metal in terms of bonds between nearest neighbors (34). To account for conduction, Pauling assumed that adjacent pairs have equal but opposite charges. Extending the concept, Anderson (35) has proposed that the superconducting oxides can be described similarly. Thus one can imagine the situation in or near the basal plane in $\mathrm{YBa}_{2} \mathrm{Cu}_{3} \mathrm{O}_{7-\delta}$ wherein the possibility of the bond between copper and oxygen can "resonate" among the two Cu1-01 and the two Cu1-04 pairs. Zou and Anderson (36), using this model, wrote the Hamiltonian such that a given site can be either empty, occupied by an electron with spin up, occupied by an electron with spin down, or doubly occupied. They recognized that the current is given by the time rate of polarization at the site:

$$
\mathrm{j}_{1}=\mathrm{dP} / \mathrm{dt}
$$

Following this, Anderson and Zou (22) evaluated the magnitude of the elastic scattering rate from the integral of the product-probabilities of vacancy and occupancy:

$$
\int f(\varepsilon)[(1-f(\varepsilon)] d \varepsilon
$$

which yields a scattering rate proportional to temperature. Thus, they concluded that the RVB description is consistent with a linear $\rho(T)$ with a high precision if $N$ in

$$
\sigma=\frac{N e^{2} \tau}{m}
$$

is independent of temperature. 
Using the measurements of the anisotropic conductivity of a single crystal of $\mathrm{YBa}_{2} \mathrm{Cu}_{3} \mathrm{O}_{7-\delta}$ made by Tozer et al. (37), Anderson and $\mathrm{Zou}$ concluded that the resistivity in both the $a-b$-plane and the c-direction can be fitted by the equation:

$$
\rho=\mathrm{AT}^{-1}+\mathrm{BT} \text {. }
$$

Hagen et al. (38) measured the resistivity of seven single crystals and reported that the resistivity could be fitted by this equation. In both of these measurements, the crystals became superconducting near $90 \mathrm{~K}$ for both directions.

To compare the equation proposed in these studies with the others, equation (4) with a constant term has been used, and rather than resistivity, conductivity has been used because it appears to be the more fundamental. Thus, the shape of the proposed equation for the conductivity vs. temperature for the RVB model is shown in Figure 1.* The curve has a maximum at $\mathrm{T}_{\mathrm{m}}=\sqrt{\mathrm{A} / \mathrm{B}}$ and an inflection at $\sqrt{3 \mathrm{~T}_{\mathrm{m}}}$.

\section{Kondo}

On the basis of s-d orbital electron interactions in dilute magnetic alloys, Kondo (23) derived the dependence of the scattering of conduction electrons on temperature. With both lattice resistivity and the factor for the s-d scattering, the equation proposed for the variation of resistivity with temperature is:

$$
\rho=\alpha \ln T+\beta T+\gamma
$$

This $\rho(T)$ has a minimum, which is frequently cited as evidence for the Kondo effect.

\footnotetext{
${ }^{*}$ For comparisons the data reported by Kini et al. (39) for sample AK-II-75 have been used. The parameters have been derived through least squares' analyses.
} 
Peng et al. (24) applied this equation to the resistivity measured in ceramic $\mathrm{Nd}_{2-\mathrm{x}} \mathrm{Ce}_{\mathrm{x}} \mathrm{CuO}_{4-\delta}$. The equation has a maximum in $\sigma(\mathrm{T})$ at $\mathrm{T}_{\mathrm{m}}=\alpha / \beta$ and an inflection given by the equation:

$$
2-3 \mathrm{~T}_{\mathrm{i}}-\mathrm{T}_{\mathrm{m}}^{-1}+2 \mathrm{~T}_{\mathrm{i}}^{2} \mathrm{~T}_{\mathrm{m}}^{-2}+\ln \mathrm{T}_{\mathrm{i}}+\alpha^{-1} \gamma=\mathrm{O}
$$

A plot of the equation for the data reported by Kini et al. (39) is shown in Figure 2. For these data the maximum occurs at $56.83 \mathrm{~K}$ and the inflection occurs at $80.87 \mathrm{~K}$

\section{Fermi Liquid}

The Fermi liquid is defined as a material in which the scattering occurs through electron-electron interactions. In this case the electrical resistivity for a three-dimensional solid has been shown to vary as $\mathrm{T}^{2}$ (See 40 ). In a survey of the subject, Kaveh and Wiser (25) examined the variations in one and two dimensions. In the first case, they reported that the resistivity is linear in temperature; in the second case, they reported that the variation is proportional to $\mathrm{T}^{2} \ln \mathrm{T}^{-1}$. Hence, for the Fermi liquid the equation for two dimensions is

$$
\rho=p_{o}+k\left(\frac{T}{T_{f}}\right)^{2} \ln \left(T_{f} / T\right)
$$

in which $T_{f}$ is the temperature corresponding to the Fermi energy and $k$ is proportional to the reciprocal of the relaxation time, i.e., $k \propto \tau^{-1}$. The equation has a maximum at $\mathrm{T}=0$ and a minimum at $\mathrm{e}^{-1 / 2} \mathrm{~T}_{\mathrm{f}}$ and an inflection at $\mathrm{T}_{\mathrm{i}}=\mathrm{T}_{\mathrm{m}} \mathrm{e}^{-1}$. $\sigma(T)$ has a minimum at $T_{m}=0$ and a maximum at $T_{m}=e^{-1 / 2} T_{f}$ and in inflection at 


$$
\rho_{i}\left(2 \ln \frac{T_{i}}{T_{f}}+3\right)+2 k\left(\frac{T_{i}}{T_{f}}\right)^{2}\left(2 \ln \frac{T_{i}}{T_{f}}+1\right)^{2}=0 .
$$

Crusellas et al. (26) and Tuesi et al. (41) have interpreted the resistivity of $\mathrm{Nd}_{1.85} \mathrm{Ce}_{0.15} \mathrm{CuO}_{4-\delta}$ in terms of equation (7). Using the data for AK-II-75, one obtains the variation of $\sigma(\mathrm{T})$ shown in Figure 3.

4. Phonon - Coupled Electron Exchange (Srivastava).

In an attempt to explain the mechanism of electrical conductivity in zinc and copper substituted magnite, Srivastava and Srivastava (42) derived an equation for the tunneling of electrons from one site to another in a process such as $\mathrm{Fe}^{2+} \rightarrow \mathrm{Fe}^{3+}+\mathrm{e}$. For temperatures above the Verway transition, they state that

$$
\sigma=\frac{2}{\pi} N \omega_{\mathrm{ph}} \mathrm{a}^{2} \mathrm{e}^{2} \beta \operatorname{sech}^{2}\left(\beta \varepsilon_{\mathrm{p}} / 2\right)
$$

in which $\mathrm{N}$ is the number of ions in the mixed valence state, $\omega_{\mathrm{ph}}$ is the frequency of the active phonon, $a$ is the distance between ions, and $\varepsilon_{p}$ is the stabilization energy of the small polaron. At temperature below the Verway transition, they assumed that thermally activated hopping occurs.

Srivastava (27) has used this equation to interpret the conductivity of $\mathrm{YBa}_{2} \mathrm{Cu}_{3} \mathrm{O}_{7-\delta}$ with $\delta$ corresponding to that for the optimum superconductivity by estimating the parameters and by comparing the calculated conductivity with the experimental value. Applying the least squares' analysis to the data for sample AK-II-75 with this equation, one obtains the results shown in Figure 4. The equation has a maximum at

$$
\frac{k T_{m}}{2 E}=\tanh \left(\frac{E}{k T_{m}}\right)
$$


Using the value for $\mathrm{E}$, one finds that the maximum occurs at $104.30 \mathrm{~K}$; an inflection occurs near $190 \mathrm{~K}$.

5. DOS: $\sigma(\varepsilon)=$ constant for $0<\varepsilon<\mathrm{W}$, otherwise zero [Moshracholkov (28)].

In the free electron theory of metals, use is made in a series expansions of integrals of the type

$$
\int_{0}^{\infty} \alpha(\varepsilon)\left(\frac{\partial f}{\partial \varepsilon}\right) d \varepsilon
$$

derived through integrations by parts $(43,44)$. By analogy one surmises that Moshachalkov has written

$$
\sigma(\mathrm{T})=-\int_{-\infty}^{\infty} \sigma(\varepsilon)\left(\frac{\partial \mathrm{f}}{\partial \varepsilon}\right) \mathrm{d} \varepsilon
$$

and assumed that $\sigma(\varepsilon)$ [the density of states] is constant in some interval $0<\varepsilon<\mathrm{W}$ and otherwise zero. According to another publication, Moshachalkov (45) imagined a density of states in which the narrow peak corresponding to $(\partial f / \partial \varepsilon)$ is located between the conduction and valence $g(\varepsilon)$ 's. With the assumed $\sigma(\varepsilon)$, one readily finds that

$$
\sigma(T)=\sigma_{0}\left\{\left(e^{-\beta E_{f}}+1\right)^{-1}-\left(e^{\beta\left(E_{g}-E_{f}\right)}+1\right)^{-1}\right\} .
$$


in which $E_{f}=c E_{F}$ and $E_{g}=c W$ in Moshachalkov's notation. He showed that if $\mathrm{E}_{\mathrm{f}}>0$, this equation yields $\mathrm{d} \rho / \mathrm{dT}>0$ and a nearly linear variation. If $\mathrm{E}_{\mathrm{f}}<0$, $\rho$ varies nonmonotonically with a minimum and a rapidly increasing variation with decreasing temperature at the lower temperature, i.e., like that for a semiconductor. Thus, if one accepts the reality of $\mathrm{E}_{\mathrm{f}}<0$, then this equation with varying $E_{f}$ describes the experimental $\rho(T)$ 's except for the maxima at low temperatures.

Applying this equation to the data of AK-II-75 through the least squares' analysis, one obtains the curve shown in Figure 5.

\section{Anharmonic/Vibronic Electron-Phonon Coupling}

Using what they call the method of force-correlation, Schiller and Wescheke (29) have calculated the resistivity of $\mathrm{YBa}_{2} \mathrm{Cu}_{3} \mathrm{O}_{7}$ for strong anharmonic vibrations interacting with electrons. For weak scattering, they find

$$
\mathrm{R}=\frac{3}{4 \pi^{5}} \frac{\mathrm{x}^{2} \mathrm{sa}}{\hbar \mathrm{e}^{7} \rho_{\mathrm{e}}^{2} \rho_{\mathrm{D}} \overline{\mathrm{v}}_{\mathrm{f}}^{2}} \frac{\left\langle\mathrm{J}_{\mathrm{s}}^{2}\right\rangle}{\left(\mathrm{e}^{\beta h \Omega}+1\right)}
$$

in which $\mathrm{x}_{\mathrm{sa}}=$ "half the distance between the two minima in the double-well potential." $<\mathrm{J}_{5}{ }^{2}>$ is electron-ion matrix element; $p_{e}$ is the density of conduction electrons; $\rho_{D}$ is the density of double-well potentials; $\bar{v}_{f}$ is the mean value of the Fermi velocity; and the $\Omega$ is the energy of the soft rotational mode. Applying this equation through a least squares' analysis to the data for AK-II-75, one obtains the results shown in Figure 6. 
7. DOS: $g(\varepsilon)=0$ for $0<\varepsilon<\mathrm{E}_{\mathrm{f}}$, otherwise constant: $\sigma\left(T ; \mathrm{E}_{\mathrm{f}}, \mathrm{E}_{\mathrm{g}}, \mathrm{n}\right)$.

In principle all the electrical properties of a solid are derivable from the electronic structure. And again, in principle the electronic structure is derivable from the band theory calculations. The difficulty is that the calculations depend on so many assumptions and approximations that such calculations are quite removed from reality. To circumvent these limitations, it seems plausible to accept only the basic elements to ascertain what can be reliably predicted. Toward this end, one recognizes that the electronic structure of the bound states can be obtained from X-ray and UV photoelectron-spectroscopy. Thus, Steiner et al. (46) have obtained the photoelectronic structure attributable to $\mathrm{Cu}(3 \mathrm{~d})$ and $\mathrm{O}(2 \mathrm{p})$ orbital electrons in $\mathrm{YBa}_{2} \mathrm{Cu}_{3} \mathrm{O}_{7-\delta}$ and further, have shown that the intensity at the Fermi level is extremely small. Several calculations of the band structure have been accomplished. As is obvious in what follows, it is not necessary to engage in a detailed study of the variously reported band-structure calculations. Rather it seems plausible to assume only the gross features, recognize that the existence of the conduction and valence bands and then ascertain what can be predicted from such. But above all else, it is essential to recognize that $\mathrm{kT}<2.6 \times 10^{-2} \mathrm{eV}$ and that the usual situation wherein the conduction electron density is so high $\left(10^{22} \mathrm{~cm}^{-3}\right.$ or more) that it forces the Fermi energy to values considerably in excess of $\mathrm{kT}$ does not apply.

Phenomenologically, one can write that the current density is given by

$$
\mathrm{j}=\mathrm{Nev}_{\mathrm{d}}
$$

in which $\mathrm{N}$ is the density of carriers of charge $e$ and $\mathrm{v}_{d}$ is the mean drift velocity in the electric field. Using the equation of motion for a free electron and defining $\bar{\tau}$ as the average time between collisions, one finds for the conductivity 


$$
\sigma=\frac{j}{E}=\frac{\mathrm{Ne}^{2} \bar{\tau}}{\mathrm{m}}
$$

In a more rigorous derivation for $\sigma$, one cannot separate the functional dependencies of $\mathrm{N}$ and $\tau$ on energy, of course (44, p. 183-4). However, one can write that

$$
N=\int_{0}^{\infty} g(\varepsilon) f(\varepsilon) d \varepsilon
$$

without any assumption such as the value of $\mathrm{E}_{\mathrm{f}}$ in relation to $\mathrm{kT}$. If in equation (17) one defines some average $g(\varepsilon)$, then one can write that

$$
N=\left\langle g(\varepsilon)>\int_{0}^{\infty} f(\varepsilon) d \varepsilon\right.
$$

where $\langle g(\varepsilon)>$ is defined through equations (17) and (18). Without loss of rigor, one can divide the integration into intervals:

$$
N=\left\langle g_{1}(\varepsilon)>\int_{0}^{E_{f}} f(\varepsilon) d \varepsilon+\left\langle g_{2}(\varepsilon)>\int_{E_{f}}^{E_{f}} f(\varepsilon) d \varepsilon+\left\langle g_{3}(\varepsilon)>\int_{E_{g}}^{\infty} f(\varepsilon) d \varepsilon\right.\right.\right.
$$

For the first interval one can write

$$
\mathrm{N}=<\mathrm{g}_{1}(\varepsilon)>\ln \left[1+\mathrm{e}^{-\beta\left(\varepsilon-\mathrm{E}_{f}\right)}\right]_{0} a_{=}=\left\langle\mathrm{g}_{1}(\varepsilon)>\mathrm{kT} \ln \left(\frac{\mathrm{e}^{\beta E_{\mathrm{f}}+1}}{2}\right)\right.
$$

This expresses a fundamental characteristic of Fermi-Dirac statistic. To the extent that a physical property is described by $\mathrm{N}\left(\mathrm{T} ; \mathrm{E}_{\mathrm{f}}\right)$, the property is determined 
by the Fermi-Dirac distribution. If one assumes that $\left\langle g_{1}(\varepsilon)>\right.$ for the interval $\mathrm{E}_{\mathrm{f}} \overline{<\varepsilon} \overline{<} \mathrm{E}_{\mathrm{g}}$ is zero then one finds that

$$
N\left(T ; E_{f}, E_{g}\right)=<g_{1}(\varepsilon)>k T \ln \left(\frac{e^{\beta E_{f}}+1}{2}\right)+<g_{3}(\varepsilon)>k T \ln \left(e^{-\beta\left(E_{\gamma}-E_{\vartheta}\right)}+1\right)
$$

or for simplicity if one assumes that $\left\langle\mathrm{g}_{1}(\varepsilon)\right\rangle=\left\langle\mathrm{g}_{3}(\varepsilon)\right\rangle$,

$$
N\left(T ; E_{f}, E_{g}\right)=k T \ln \left\{\frac{\left(e^{\beta} E_{f}+1\right)\left(e^{-\beta(E} E_{r} E_{r}+1\right)}{2}\right\} .
$$

After the fact, one can now examine $\bar{\tau}(T)$.

The relaxation time, $\tau(T)$, depends on, of course, the scattering mechanism. For electron-phonon scattering in three dimensions (See 47), it is usually assumed to be proportional to $\mathrm{T}^{-1}$ and for electron-electron scattering, frequently it is assumed to be proportional to $\mathrm{T}^{-2}$, unless it is modified as indicated above in the two-dimensional Fermi liquid. For the present purpose, it is adequate to assume that $\bar{\tau} \propto \mathrm{T} \sim{ }^{*}$ Hence, one finds that

$$
\sigma\left(T ; E_{f}, E_{g_{1}} n\right)=C T^{(1-n)} \ln \left\{\frac{\left(e^{\beta E_{f}}+1\right)\left(e^{-\beta\left(E_{8}-E_{f}\right)}+1\right)}{2}\right\}
$$

\footnotetext{
*For the temperature range of interest $(100-300 \mathrm{~K}$ ), a least squares' analysis of a comparison of the equation (7) for the two-dimensional Fermi liquid and a resistivity which depends on BT ${ }^{\mathrm{n}}$, one finds the result (using intervals of $25 \mathrm{~K}$ ): $\mathbf{T}$

300

200

100

Percent difference

$-168 \times 10^{-2}$

$-2.18 \times 10^{-2}$

$2.08 \times 10^{-1}$

Hence, within the precision at present obtained for $\rho(\mathrm{T})$ or $\sigma(\mathrm{T})$ the two, Fermi liquid and $\mathrm{BT}^{\mathrm{n}}$, are equivalent with $n=2.17$.
} 
Thorn and Thorn (31) have used this equation to interpret the variation of conductivity with temperature and composition for $\mathrm{YBa}_{2} \mathrm{Cu}_{3} \mathrm{O}_{7-\delta}$ and $\mathrm{YBaCu}_{3(1-\mathrm{x})} \mathrm{Fe}_{\mathrm{x}} \mathrm{Cu}_{3} \mathrm{O}_{7-\delta}$ and of $\sigma(\mathrm{T})$ for $\mathrm{La}_{1.85} \mathrm{Ba}_{0.15} \mathrm{CuO}_{4}$ and $\mathrm{BaPb}_{0.8} \mathrm{Bi}_{0.2} \mathrm{O}_{3}$. In this investigation, they found $E_{f}$ of the order of $10^{-4}-10^{-3} \mathrm{eV}, \mathrm{E}_{\mathrm{g}}$ of the order of $10^{-}$ 2 , and $\mathbf{n}$ of the order of 2 . The structures of the variation of $\sigma(T)$ 's for a fixed value of $E_{g}$ and $N$ and for varying $E_{f}$ are shown in Figure 7. Of particular interest is the fact that the equation predicts the minimum and the rapid increase in $\sigma$ as $\mathrm{T}$ is decreased in cases in which the diamagnetic susceptibility is extremely small or undetectable (30) such as observed by Beyer et al. (48), Takagi et al. (49), Mazaki et al. (50) and Senago and Oonu (51). In addition, the equation is consistent with a linear variation of $\rho(T)$, a variation which is frequently cited for the case with the highest superconducting temperature.

Within a precision of 0.1 percent or less, the linear variation of the $\rho(T)$ is contained in equation (23). To illustrate this, the data for $\rho(T)$ reported by Kini et al. were fitted to a linear equation through a least squares' treatment. The result is

$$
\rho=(4.28 \pm 0.12) \times 10^{-1}+(2.208 \pm 0.079) 10^{-3} \mathrm{~T}
$$

with a chi square per degree of freedom of $2.53 \times 10^{-1}$ and a likelihood of 78 percent. The values for $\sigma\left(=\rho^{-1}\right)$ obtained from the least squares' equation were then used to fit equation (23) through a least squares' analysis. The fitted values for the equation for $\rho(T)$ are within 0.013 percent or less of the linear least squares' values for $\rho(T)$. The point at $91 \mathrm{~K}$ represents the onset of the superconducting state. 


\section{Small Polaron}

In Maxwell's equation,

$$
\text { curl } H=\frac{1}{c} \frac{d E}{d t}+\frac{4 \pi}{c} \frac{d P}{d t}+\frac{4 \pi}{c} J
$$

one can recognize, as Condon and Odishaw (52) have, two currents: (1) J, which is attributable to charge that moves over many atomic dimensions, i.e., "free" charges, and (2) a current attributable to movement over atomic dimensions and associated with the time rate of change of electronic polarization. Accordingly, the rate of transfer of charge via polarons is proportional to the time rate of change of the dipole moment [See Böttger \& Bryksin (33)]:

$$
\mathrm{j}=\frac{1}{\Omega} \frac{\mathrm{dD}(\mathrm{t})}{\mathrm{dt}} .
$$

Using this expression and a Hamiltonian containing electron-phonon coupling, Böttger \& Bryksin developed further the small polaron theory originally presented by Holstein (32). One of the most significant features of the theory is the fact that the total conductivity is the sum of that for two mechanisms: one attributable to thermally activated hopping between sites and dominant at high temperatures and one attributable to narrow-band tunneling which dominates at low temperature:

$$
\sigma=\sigma_{\mathrm{h}}+\sigma_{\mathrm{t}}
$$

The precise forms of $\sigma_{\mathrm{h}}$ and $\sigma_{\mathrm{t}}$ depend on the magnitude of the parameters, but for the present the following is accepted 


$$
\sigma=C_{h}(1-f) T^{-3 / 2} E^{-1 / 2} e^{-\beta E}+C_{t} T^{-1} g^{1 / 2}[\operatorname{csch}(\beta \omega)]^{1 / 2} e^{-8 \operatorname{csch}(\beta \omega)}
$$

In this equation $f$ is the Fermi-Dirac distribution, $E$ is the activation energy for thermal hopping, $g$ is a measure of the electron-phonon coupling, and $\omega$ is an effective frequency of vibration of the longitudinal optical phonon along the direction of transfer.

The structures of the variations of the $\sigma(T)$ 's for the small polaron are the same to those for the averaged valence-band (conduction band) [see equation (23)]. The essential difference is that the two parts of equation (28) are approximations which describes quantitatively the high and low temperature ranges, but they do not necessarily do so well in the intermediate range where the two are comparable (Fig. 9).

Thorn (53) has shown that the small polaron model describes quantitatively the measurements of $\rho(T)$ by Beyer et al. (48) for a sample having an extremely small diamagnetic susceptibility.

\section{Comparisons and Remarks}

All of the equations cited above have been used in least squares' analyses of the values for $\rho(\mathrm{T})$ reported for $\mathrm{YBa}_{2} \mathrm{Cu}_{3} \mathrm{O}_{7-\delta}$, sample AK-II-75 by Kini et al. In Table II are listed the experimental values for $\sigma(\mathrm{T})$ obtained from the curve reported by Kini et al. For each equation, there are listed the percent deviations of the least squares' values from the experimental values. Also listed are the values for chi square/degree of freedom. It is obvious that the values for equation (I-7) are the smallest and that the values for the small polaron equation are also significantly smaller than the values for the other equations. 
The equations for RVB, Kondo, conduction-band/valence-band, and small polaron all contain two terms: one for a "semiconductor" and one for a "metal." Hence, there is a recondite awareness and consequently an implication in all the elementary conceptualizations of the existence of two mechanisms. It is particularly significant to recognize that equations (I-7) and (I-8) predict that the apparently simple variation such as that reported for AK-II-75 and that the frequently cited linear variation in $\rho(T)$ consists of the two parts, and thereby they reveal a generally unrecognized, but possible, feature of conductivity in the normal state.

The additive terms for $\rho(T)$ imply sequential events; the additive terms for $\sigma(T)$ imply simultaneous events. Of the two, $\sigma(T)$ must be considered to be the more fundamental, because $\rho(T)$ 's are based only on scattering.

Table II. Comparisons of Application of Equations in Table I to Conductivity of $\mathrm{BBa}_{2} \mathrm{Cu}_{3} \mathrm{O}_{7-\delta}$, AK-II-75.

$\mathrm{T}$

$\begin{array}{rrrrrrrrrr} & & \text { RVB } & \text { K } & \text { FL } & \text { S } & & & & \text { SP } \\ & & 1^{\mathrm{c}} & 2 & 3 & 4 & 5 & 6 & 7 & 8 \\ 250 & 1.000 & -0.50 & -0.34 & -0.10 & 2.89 & -0.32 & -7.50 & 0.04 & 0.09 \\ 225 & 1.0752 & 0.06 & 0.05 & 0.03 & 1.92 & 0.03 & -3.05 & 0.02 & -0.01 \\ 200 & 1.1547 & 0.21 & 0.11 & -0.04 & 0.50 & 0.08 & 0.79 & -0.18 & -0.22 \\ 175 & 1.2451 & 0.53 & 0.43 & 0.29 & -0.64 & 0.41 & 4.13 & 0.12 & 0.13 \\ 150 & 1.3332 & 0.03 & 0.02 & 0.01 & -2.13 & 0.05 & 5.15 & 0.01 & 0.06 \\ 125 & 1.4226 & -0.60 & -0.50 & -0.38 & -2.50 & -0.44 & 3.02 & -0.03 & -0.06 \\ 100 & 1.5261 & 0.22 & 0.21 & 0.18 & 2.12 & 0.16 & -3.40 & 0.006 & 0.01 \\ & & & & & & & & & \\ \text { ChiSq/DF x 104 } & 0.40 & 0.26 & 0.13 & 9.0 & 0.21 & 38 & 0.023 & 0.037 \\ \text { No. of Parameters } & 33 & 3 & 1 & 2 & 3 & 2 & 4 & \\ \text { for } \sigma_{n}(\mathrm{~T}) & & & & & & & & \end{array}$


aObtained from curve for AK-II-75 published by A. M. Kini, et al. (39).

bThe number of significant figures does not represent the precision of reading the values from the curve but merely the reciprocals of the values $\left(p^{-1}\right)$ which were obtained.

cThese numbers correspond to the equations numbered in Table $\mathrm{I}$.

\section{Discussion}

Several measurements of the resistivities (conductivity) of $\mathrm{YBa}_{2} \mathrm{Cu}_{3} \mathrm{O}_{7-\delta}$ as functions of temperature and composition (48-51), and in a few of the cases, the dependency of the diamagnetic susceptibility on temperature and composition for the same samples used to obtain the resistivities have been reported. All of these measurements display the same evolution of the structures of $\sigma(T)$ 's as the compositions are varied. For compositions with $\delta \sim 0.1, \rho(T)$ above the superconducting temperature increases monotonically and nearly linearly with increasing temperature. For compositions with $\delta$ near 0.7 (or near $\mathrm{YBa}_{2} \mathrm{Cu}_{3} \mathrm{O}_{6}$ ) the $\rho(\mathrm{T}$ )'s increase with decreasing temperature as expected for a semiconductor, but for $\delta$ near 0.7 a maximum occurs and $\rho(\mathrm{T})$ decreases rapidly as the temperature is decreased further. Measurements of the diamagnetic susceptibilities $\chi(T, \delta)$ for $\delta$ $<0.6$ show that at a fixed $\delta$ the usually observed signoidal variation occurs, but as $\delta$ increases the asymptotic value becomes more positive until finally it is zero at all the temperatures for $\delta$ near 0.7. The systematic variations in both $\rho(T, \delta)$ and $\chi(T, \delta)$ have been reported particularly by Takage et al. and Mazaki et al. Selecting some representative cases, one obtains variations of $\sigma(T, \delta)$ shown in Figure 10.

This pattern appears to be well established, but it appears to be little recognized, and its significance has not been generally appreciated. DeJongh (54) has recognized part of it and has discussed it qualitatively in terms of concepts presented by Mott and Kaveh (55) and the small polaron. But one significant feature not discussed by de Jongh is the rapid decrease in $\rho(T)$ below the 
maximum partly perhaps because it is obscured by the onset of the residual material in the superconducting state. However, the measurements by Mazaki et al. with $\delta=0.31$ seem to establish that this rapid decrease is not primarily attributable to the presence of superconducting material.

One must question, of course, whether the systematic variations in $\rho(T, \delta)$ obtained by Mazaki et al and Takagi et al. can be attributable to some particular characteristic of the ceramic material. Studies with single crystals yield $\rho(T)$ 's in the $a b$ plane with $\frac{\mathrm{dR}}{\mathrm{dT}}>0$ and in the $c$ direction with the semiconductive variation $\frac{\mathrm{d} R}{\mathrm{dT}}<0$. Further, Mosolov (56) has shown that over a limited range of temperatures $\left(920-960^{\circ}\right)$ for the sintering, the shape of $\rho(T)$ does not vary, although the resistivity decreases by a factor of four.

Among all the equations listed in the tables and illustrated in figures, it is apparent from the inspections of the variations of $\sigma(T)$ that only the ones for $\sigma(T$; $\left.E_{f}, E_{g}, n\right)$ and for the small polaron of Holstein can describe quantitatively the observed experimentally established pattern.

\section{Acknowledgments}

I express my thanks to J. M. Williams for the opportunity to conduct this study and my appreciation of the efforts of V. Bowman and J. Bormet for processing the manuscript. Work at Argonne National Laboratory is sponsored by the U.S. Department of Energy, Office of Basic Energy Sciences, Divisions of Materials and Chemical Sciences, under contract W-31-109-ENG-38. 


\section{References}

1. X. L. Lei, J. Phys. C, Solid State Phys., 21, L83 (1988).

2. J. Heremans, D. T. Morelli, G. W. Smith, and S. C. Strite, III, Phys. Rev. B, Condens. Matter, 37, 1604 (1988).

3. S. A Koz'min, V. I. Kaidanov, and G. Leising, Sov. Phys. - Solid State, 30, 1703 (1988).

4. H. C. Yang, M. H. Hsieh, H. H. Sung, C. H. Chen, H. E. Horng, Y. S. Kan, N. C. Chen, and J. C. Jao, Phys. Rev. B, Condens. Matter, 39, 9203 (1989).

5. H. Kumar, Phys. Rev. B, Condens. Matter, 40, 844 (1989).

6. E. S. Hellman, Phys. Rev. B, Condens. Matter, 39, 9604 (1989).

7. T. K. Chaki and M. Rubenstein, in Superconductivity and Its Applications, Proceedings of the Second Annual Conference on Superconductivity and Its Applications, Elsivier, p 49, 1988.

8. C. P. Enz, Mod. Phys. Lett. B, 3, 919 (1989).

9. J. C. Phillips, Phys. Rev. B. Condens. Matter, 40, 7348 (1989).

10. I. F. Itskovich and R. I. Shekhter, Sov. J. Low Temp. Phys., 15, 1138 (1989).

11. J. H. Kim, K. Levin, R. Wentzcovitch, and A. Auerbach, Phys. Rev. B, Condens. Matter, 40, 11378 (1989).

12. L. Forro, C. Ayache, J. Y. Henry, and J. Rossat-Mignod, Phys. Scr., 41, 365 (1990).

13. J. R. LaGraff, P. D. Han, and D. A. Payne, Physica C, 169, 355 (1990).

14. I. B. Ioffe and P. B. Wiegmann, Phys. Rev. Lett., 65, 653 (1990).

15. B. Sundquist and B. M. Andersson, Solid State Commun., 76, 1019 (1990).

16. I. B. Ioffe and G. Kotliar, Phys. Rev. B, Condens. Matter, 42, 10348 (1990).

17. D. Y. Xing and M. Liu, Phys. Rev. B, Condens. Matter, 43, 3744 (1991).

18. Z. Tesanovic, in AMSANTS'90. Advances in Materials Science and Application of High Temperature Superconductors, NASA, p 373, 1991. 
19. G. M. Carneiro, J. Phys. Condens. Matter, 3, 3671 (1991).

20. D. Izzo, Phys. Rev. B., Condens. Matter, 44, 85 (1991).

21. Q. Si and K. Levin, Phys. Rev. B, Condens. Matter, 44, 4727 (1991).

22. P. W. Anderson and Z. Zou, Phys. Rev. Lett., 60, 132 (1988).

23. J. Kondo, Solid State Physics (Edited by F. Seitz, D. Turnbull and H. Ehreneich) Academic Press, N.Y., 23, 183 (1969).

24. J. L. Peng, R. N. Shelton, and H. B. Radousky, Phys. Rev. B, 41, 187 (1990).

25. M. Kaveh and N. Wiser, Adv. Phys., 33. 257 (1984).

26. M. A. Crusellas, J. Fontcuberta, S. Piñol, T. Grenet, and J. Beille, Physica C,180, 313 (1991).

27. C. M. Srivastava, Physica C, 176, 481 (1991).

28. V. V. Moshchalkov, Solid State Commun., 73, 777 (1990).

29. W. Schiller and K. Weschke, Phys. Stat. Sol. (b), 160, 297 (1990)

30. R. J. Thorn, J. Phys. Chem. Solids, 48, 355 (1987); Chemistry of High Temperature Superconductors (Edited by D. L. Nelson, M. S. Whittingham, and T. G. George, American Chemical Society, Washington D.C, p 25, (1987).

31. R. J. Thorn and C. E. Thorn, J. Phys. Chem. Solids, 50, 153 (1989).

32. T. Holstein, Ann. Phys. (N.Y.) 8, 325, 343 (1959).

33. H. Böttger and V. V. Bryksin, Hopping Conduction in Solids, VCH Publishers, Deerfield Beach, Fl, 1985.

34. L. Pauling, Nature of the Chemical Bond, Cornell University Press, 1968.

35. P. W. Anderson, Science, 235, 1196 (1987).

36. Z. Zou and P. W. Anderson, Phys. Rev. B, 37, 627 (1988).

37. S. W. Toser, A. W. Kleinsasser, T. Penney, D. Kaiser, and F. Holtzberg, Phys. Rev. Lett., 59, 1768 (1987). 
38. S. J. Hagen, T. W. Jing, Z. Z. Wang, J. Horvath, and H. P. Ong, Phys. Rev. B, 37, 7928 (1988).

39. A. M. Kini, U. Geiser, H.-C. Kao, K. D. Carlson, H. H. Wang, M. R. Monaghan, and J. M. Williams, Inorg. Chem., 26, 1834 (1987).

40. E. Abraham, Phys. Rev. B, 95 (1954) 839; See also D. Pines, Solid State Physics (Edited by F. Seitz and D. Turnbull) Academic Press, N. Y., 1, 367 (1955).

41. C. C. Tuesi, A. Gupta, and G. Koren, Physica C, 161, 415 (1989).

42. G. Srivastava and C. M. Srivastava, Phys. Status Solidi B, 103, 665 (1981).

43. F. Seitz, The Modern Theory of Solids, McGraw Hill Book Co., Inc, New York, p 147, 1940.

44. F. J. Blatt, Physics of Electronic Conduction in Solids, McGraw Hill Book Co., Inc., New York, pp. 59-61, 1968.

45. V. V. Moshchalkov, Physica C, 156, 473 (1988).

46. P. Steiner, V. Kisinger, I. Sander, B. Siegwart, S. Hüfner, and C. Politis, Z. Phys. B., Cond. Matter, 67, 19 (1987).

47. L. J. Sham and J. M. Zimer, Solid State Physics (Edited by F. Seitz and D. Turnbull) 15, p. 289, Academic Press, N. Y., 15, 289 (1963).

48. R. Beyers, E. M. Engler, P. M. Grant, S. S. P. Parkin, G. Lim, M. L. Ramirez, K. P. Roche, J. E. Vazquez, V. Y. Lee, and R. D. Jacowitz, B. T. Aku, T. M. Gür, and R. A Huggins, Progress in High Temperature Superconductors (Editors, J. Heiras, R. A. Barrio, T. Akachi, J. Tagüeña) World Scientific, N. J., p 38, (1988).

49. H. Takagi, S. Uchida, H. Iwabuchi, H. Eisaki, K. Kishio, K. Kitazawa, K. Fueki, and S. Tanaka, Physica B, 148, 349 (1987).

50. D. H. Magaski, Y. Ueda, Y. Aikara, T. Kubozoe, and K. Kosuge, Jpn. J. Appl. Phys., 28, L368 (1989). 
51. K. Suenaga and M. Oonu, J. Phys. Soc. Jpn., 60, 1189 (1991).

52. E. V. Condon and H. Oldishaw, Handbook of Physics, McGraw-Hill Book Co., New York, 1987.

53. R. J. Thorn, Physica C, 190, 193 (1992).

54. L. J. de Jongh, Physica $C, 152,171$ (1988).

55. N. F. Mott and M. Kevek, Adv. Phys., 34, 329 (1985).

56. A. B. Mosolov, Superconductivity, 4, 282 (1991). 
Figure Captions

Figure 1 Results of the least squares' application of RVB equation (I-1) with constant term to data of Kini (39) $\mathrm{YBa}_{2} \mathrm{Cu}_{3} \mathrm{O}_{7-\delta}$, AK-II-75.

Figure 2 Results of the least-squares' application of Konde equation (I-12) to data of $\mathrm{Kini}$ (39), $\mathrm{YBa}_{2} \mathrm{Cu}_{3} \mathrm{O}_{7-\delta}$, AK-II-75.

Figure 3 Results of the least squares' application of Fermi liquid equation (I-3) to data of $\mathrm{Kini}$ (39), $\mathrm{YBa}_{2} \mathrm{Cu}_{3} \mathrm{O}_{7-\delta}$, AK-II-75.

Figure 4 Results of the least-squares' application of phonon-coupled exchange Srivastava (I-4) to data of Kini (39), $\mathrm{YBa}_{2} \mathrm{Cu}_{3} \mathrm{O}_{7-\delta}$, AK-II-75.

Figure 5 Results of the least squares' application of Moshchalkov (I-5) equation for DOS: $\sigma(\varepsilon)=$ const. $0<\varepsilon<W$ and otherwise 0 to data.

Figure 6 Results of the least squares' application of equation (I-6) by Schiller and Weschke for electrons coupled to unharmonic vibration to data of $\mathrm{Kini}$ (39), $\mathrm{YBa}_{2} \mathrm{Cu}_{3} \mathrm{O}_{7-\delta}$, AK-II-75.

Figure 7 Structure of $\sigma\left(T ; E_{f}, E_{g}, n\right)$ equation (23) for DOS: $g(\varepsilon)=0$ for $\mathrm{E}_{\mathrm{f}}<\varepsilon<\mathrm{E}_{\mathrm{g}}$, otherwise constant: $\sigma\left(\mathrm{T} ; \mathrm{E}_{\mathrm{f}}, \mathrm{E}_{\mathrm{g}}, \mathrm{n}\right)$ with $\mathrm{E}_{\mathrm{g}}=2.5 \times 10^{-2} \mathrm{eV}$, $\mathrm{n}=2$ and $\mathrm{E}_{\mathrm{f}}=0$ (a), $2 \times 10^{-4} \mathrm{eV}(\mathrm{b}), 5 \times 10^{-4} \mathrm{eV}(\mathrm{c}), 1 \times 10^{-3} \mathrm{eV}$ (d), $5 \times 10^{-3} \mathrm{eV}(\mathrm{e}), 1.27 \times 10^{-2} \mathrm{eV}(\mathrm{f})$; in the last case $\rho(\mathrm{T})$ is nearly linear. 


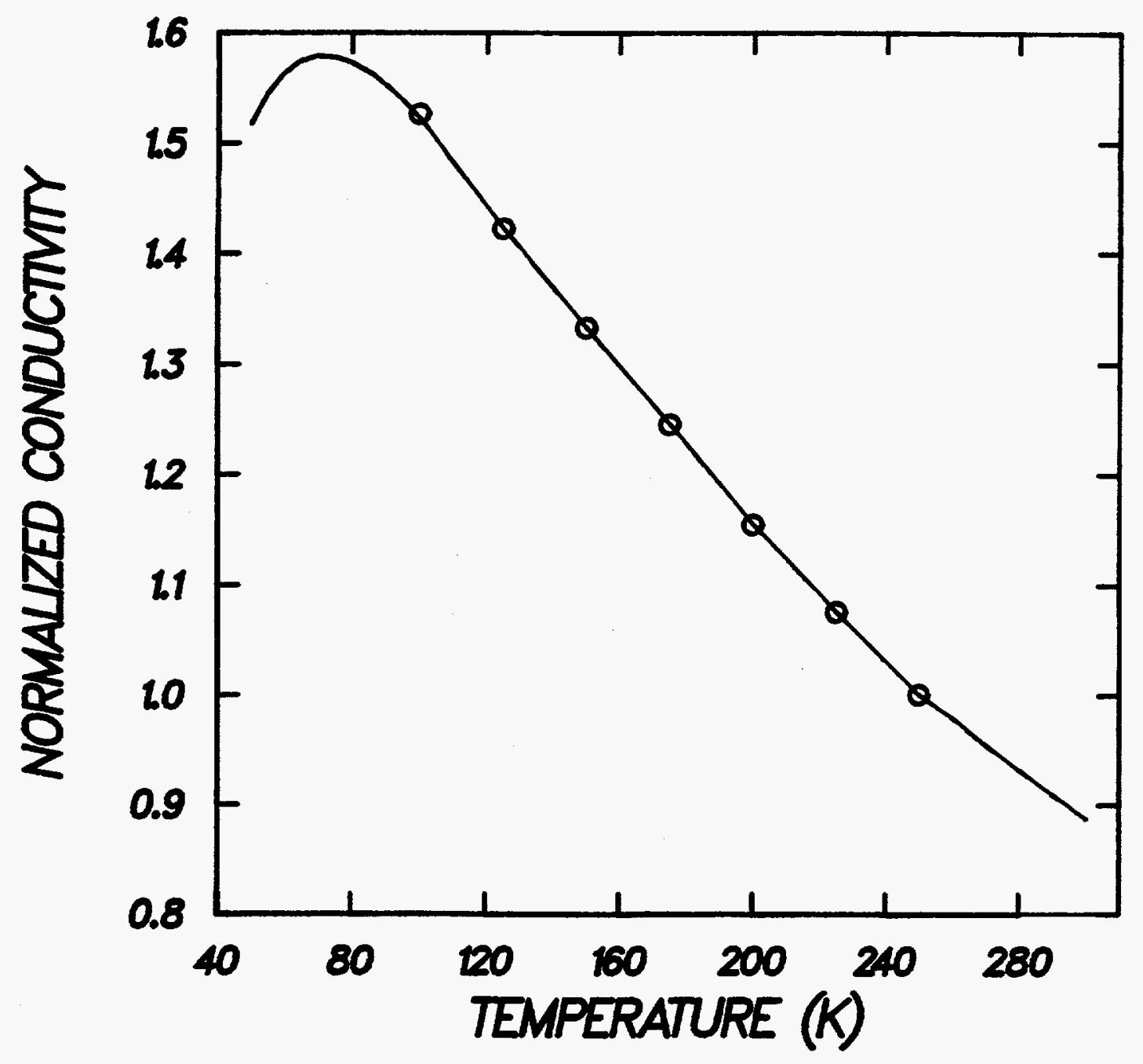

$R \cup B$ 


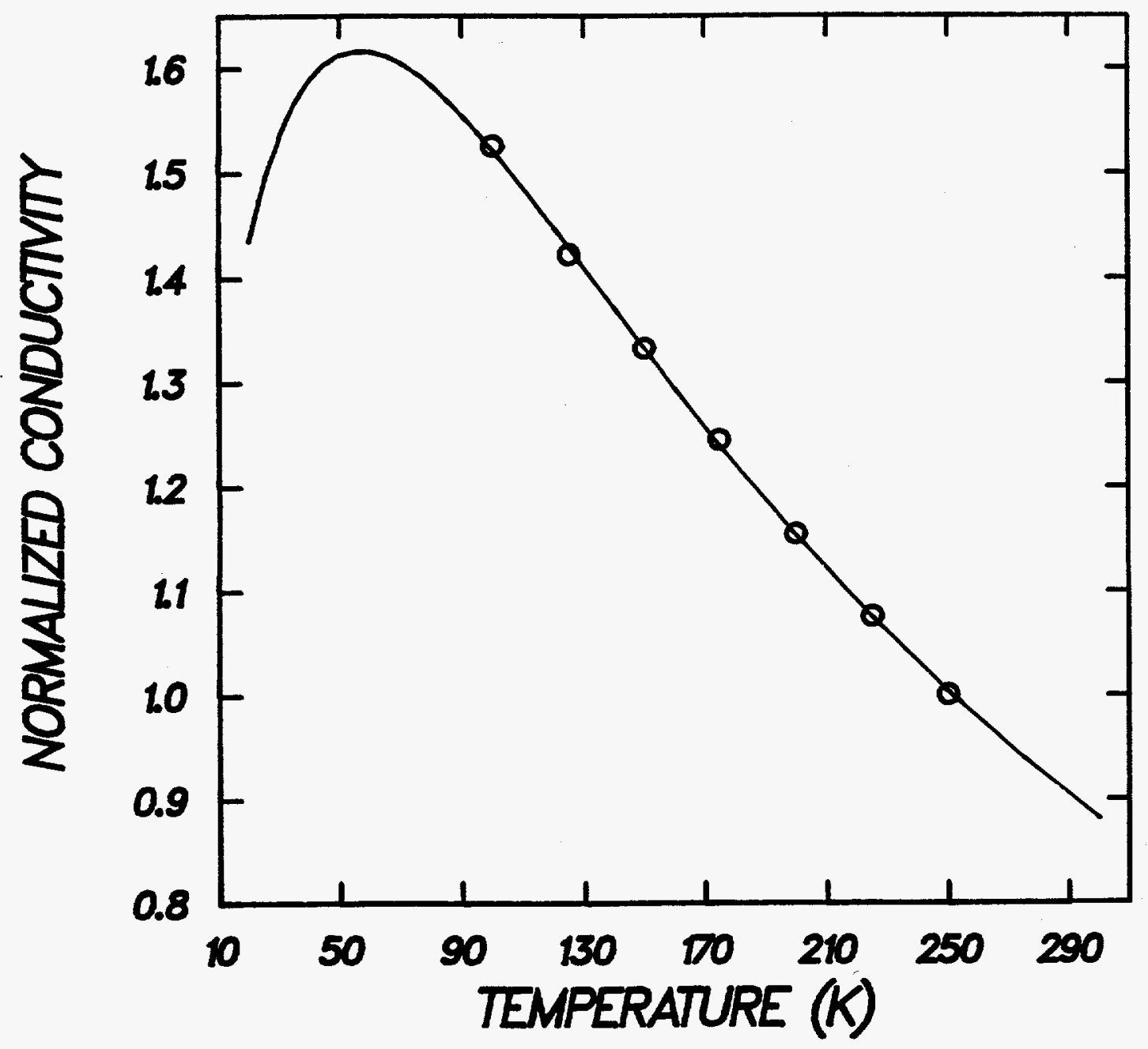




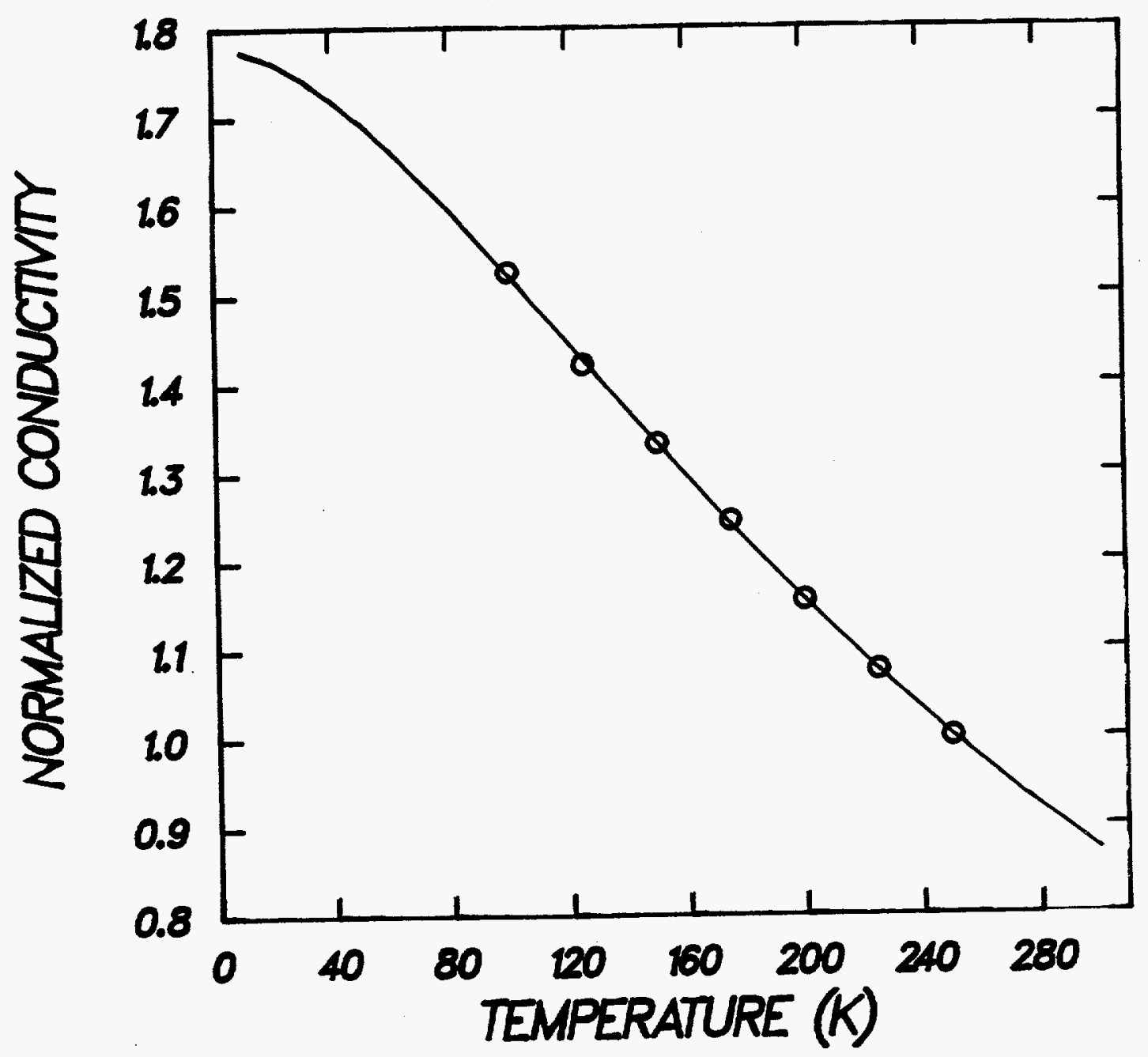

3

EERMIL LDE 


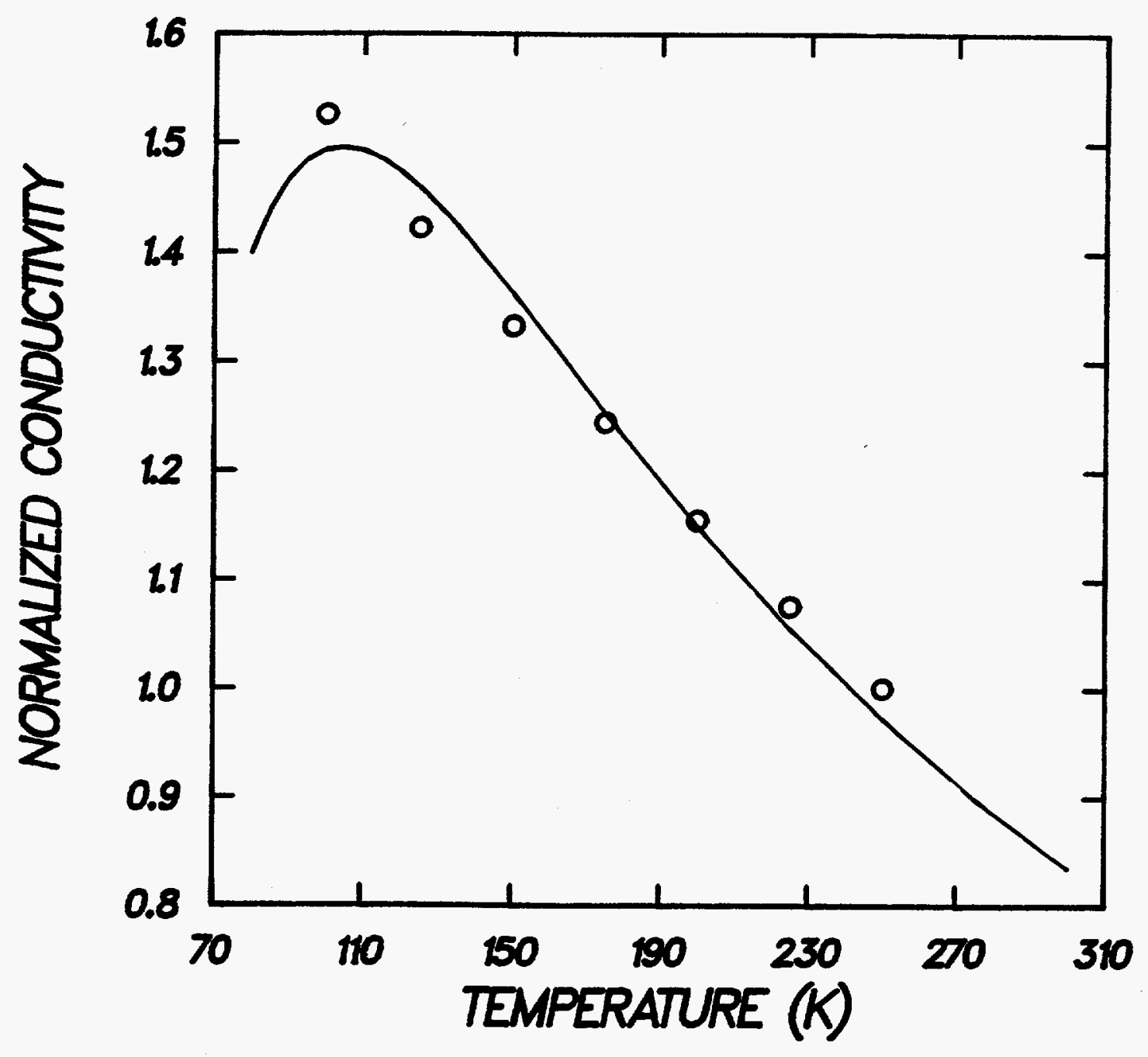




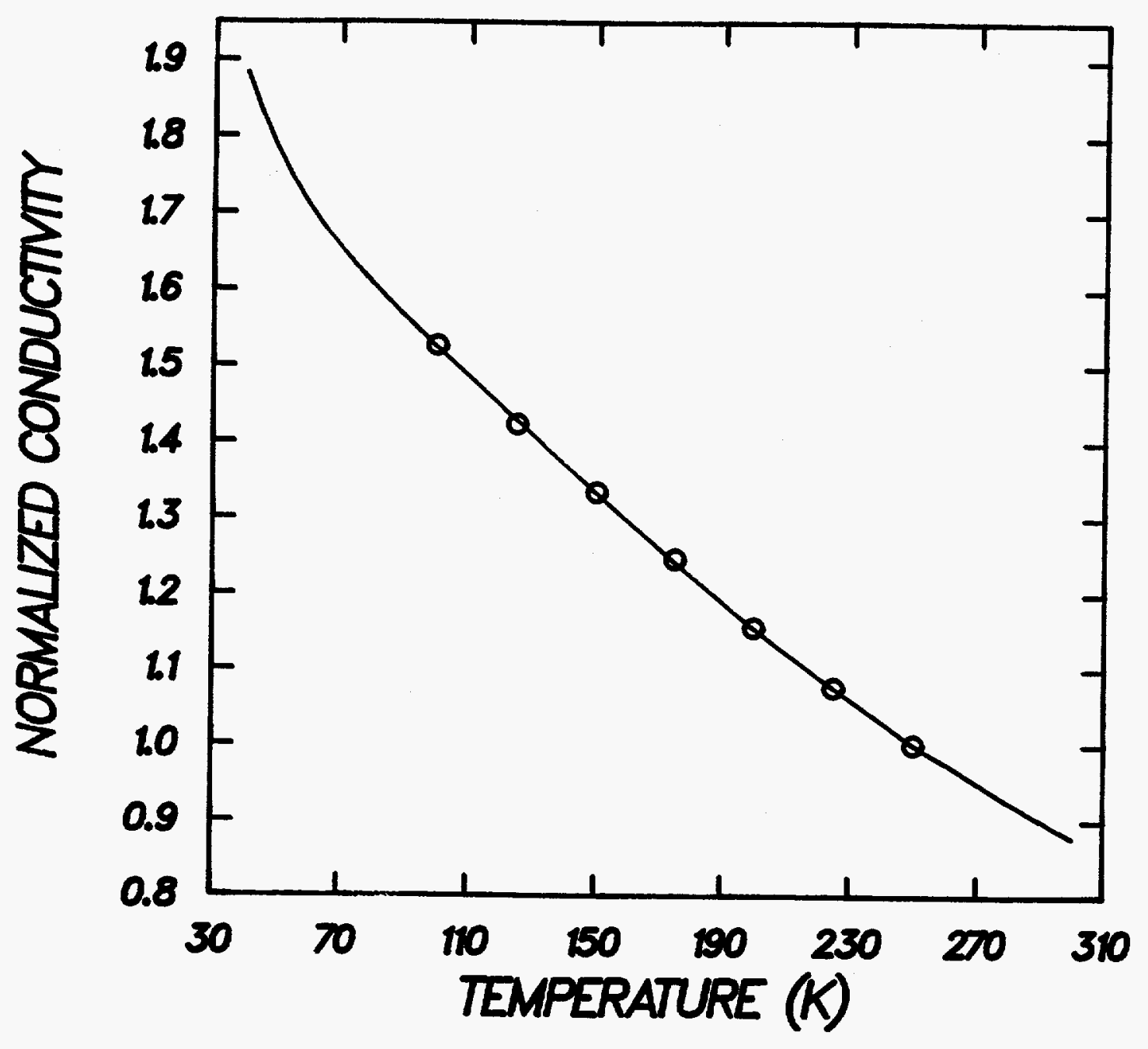

Nies! 


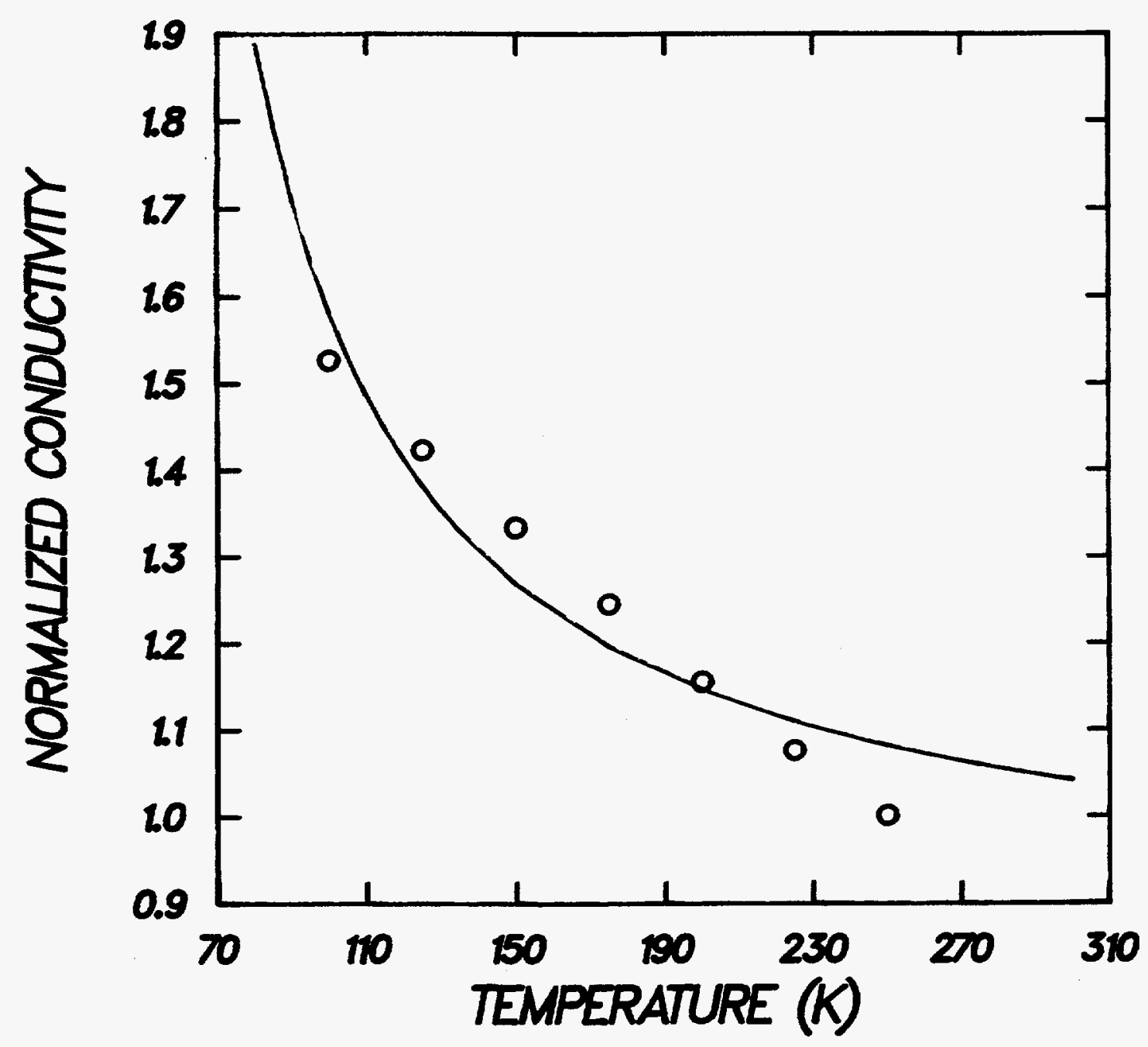




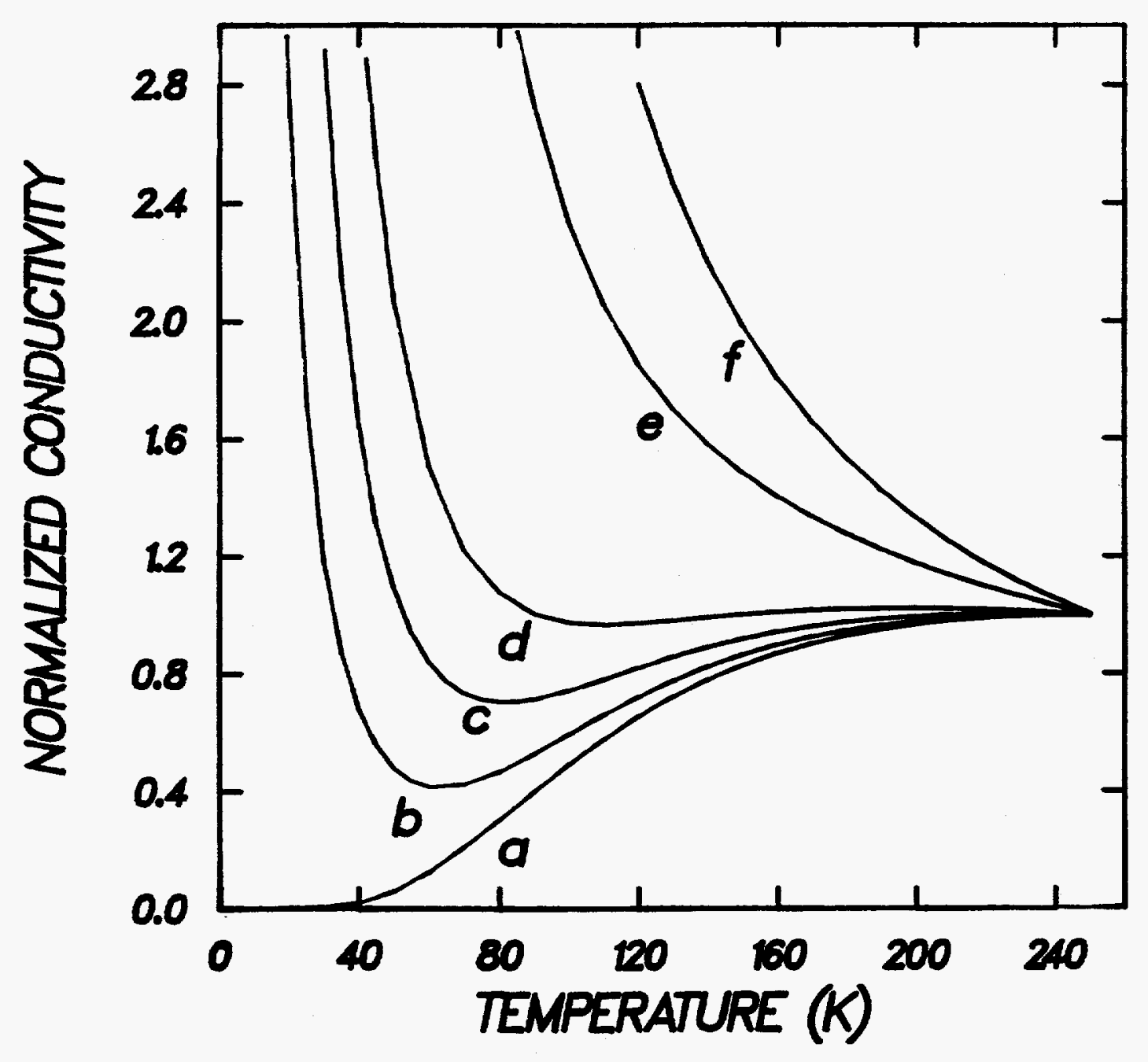

